\title{
ISPI: the infrared side port imager for the CITO 4-m telescope
}

Ronald G. Probst, Andres Montane, Michael Warner, Maxime Boccas, Marco Bonati, et al.

Ronald G. Probst, Andres Montane, Michael Warner, Maxime Boccas, Marco Bonati, Ramon Galvez, Roberto Tighe, Michael C. Ashe, Nicole S. van der Bliek, Robert D. Blum, "ISPI: the infrared side port imager for the CITO 4-m telescope," Proc. SPIE 4841, Instrument Design and Performance for Optical/Infrared Ground-based Telescopes, (7 March 2003); doi: $10.1117 / 12.461435$

Event: Astronomical Telescopes and Instrumentation, 2002, Waikoloa, Hawai'i, United States 


\title{
ISPI: the Infrared Side Port Imager for the CTIO 4-m telescope
}

\author{
Ronald G. Probst ${ }^{\mathrm{a}, \mathrm{b}}$, Andres Montane ${ }^{\mathrm{b}}$, Michael Warner ${ }^{\mathrm{b}},{\text { Maxime } \text { Boccas }^{\mathrm{b}, \mathrm{c}} \text {, Marco Bonati }}^{\mathrm{b}, \mathrm{d}}$, \\ Ramon Galvez ${ }^{\mathrm{b}}$, Roberto Tighe ${ }^{\mathrm{b}}$, Mike Ashe ${ }^{\mathrm{e}, \mathrm{f}}$, Nicole van der Bliek $^{\mathrm{b}}$, Robert Blum ${ }^{\mathrm{b}}$ \\ ${ }^{a}$ National Optical Astronomy Observatory, PO. Box 26732, Tucson AZ 85736-6732 USA* \\ ${ }^{\mathrm{b}}$ Cerro Tololo Interamerican Observatory, Casilla 603, La Serena, Chile* \\ ${ }^{c}$ Gemini Observatory Southern Operations Center, Casilla 603, La Serena, Chile \\ ${ }^{\mathrm{d}}$ California Institute of Technology Optical Observatories, MS 105-24, Pasadena, CA 91125 USA

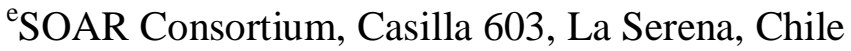 \\ ${ }^{\mathrm{f}}$ Imaginatics, Inc.
}

\begin{abstract}
The new operations model for the CTIO Blanco 4-m telescope will use a small suite of fixed facility instruments for imaging and spectroscopy. The Infrared Side Port Imager, ISPI, provides the infrared imaging capability. We describe the optical, mechanical, electronic, and software components of the instrument. The optical design is a refractive camera-collimator system. The cryomechanical packaging integrates two $\mathrm{LN}_{2}$-cooled dewars into a compact, straightline unit to fit within space constraints at the bent Cassegrain telescope focus. A HAWAII 22048 x $2048 \mathrm{HgCdTe}$ array is operated by an SDSU II array controller. Instrument control is implemented with ArcVIEW, a proprietary LabVIEW-based software package. First light on the telescope is planned for September 2002.
\end{abstract}

Keywords: infrared imaging, infrared camera, optical design, array control, instrument control

\section{INFRARED IMAGING AT CTIO IN THE LARGE TELESCOPE ERA}

The study of the growth of structure and complexity in the Universe requires high sensitivity near infrared imaging. This provides morphological and physical information essential to the study of the formation, clustering, and evolution of galaxies; the fragmentation of molecular clouds into stars; and the birth of planetary systems. While SIRTF and other space missions will provide important gains in the thermal IR, the 1-2.5 micron region is readily accessible with groundbased telescopes and is rich in relevant astrophysical phenomena. At large lookback times, the visible spectrum is redshifted into the near IR, allowing direct comparisons between the local and distant Universe. This region also offers a steep decline in dust opacity and a wealth of diagnostic spectral features from atomic and molecular gas. It is fertile ground for study of the physics of the interstellar medium, embedded stars, and planetary system formation. Objects can be readily identified and characterized by continuum emission, or by morphology and flux in characteristic strong spectral lines. Time-consuming followup spectroscopy can then be performed selectively, guided by the imaging results.

Correspondence: R. Probst, probst@noao.edu

* Operated by the Association of Universities for Research in Astronomy, Inc. under cooperative agreement with the National Science Foundation. CTIO is a division of NOAO. 
As a result of these science drivers, infrared imaging has long been a workhorse capability on the 4-m Blanco telescope of the Cerro Tololo Interamerican Observatory (CTIO). A series of infrared cameras and instrument upgrades has provided increasing fields of view at sub-arcsecond spatial resolution, keeping pace with the format growth of infrared arrays. Scientific productivity has been substantial. A continuation of this effort to take advantage of the largest currently available IR array format is a natural extension of this heritage. However, the scientific and programatic considerations for CTIO are somewhat more complex. The Gemini South 8-m telescope ${ }^{1}$ and the SOAR Consortium 4.2-m telescope ${ }^{2}$, both nearby on Cerro Pachon, will soon be providing powerful infrared facility instruments to the same user community served by CTIO. These telescopes are optimized to provide superb image quality over small fields of view. The infrared cameras under construction for them, in conjunction with adaptive optics systems, will enable high angular resolution, high sensitivity observations over arcminute to arcsecond field angles. There remains a complementary need for wide field imaging capability with large apertures. Rapid, deep coverage of wide areas is necessary, for example, to identify and trace physical structures of large extent-from outflows in nearby star forming regions to cluster structure in the population of distant galaxies. The CTIO 4-m is well suited to deep infrared imaging that emphasizes field of view. Conceived for wide-field imaging in the photographic era, investment in telescope upgrades has met the sub-arcsecond imaging performance needs of electronic sensors.

These scientific considerations set the top level requirements for a new facility IR camera: coverage of 1-2.4 microns with broadband and narrowband filters, using the largest available format at $\sim 0.5$ arcsecond per pixel resolution. Further programmatic considerations arise from CTIO's resource commitments for startup of the SOAR telescope, and for future major instrumentation activities within NOAO. These require scaling back the operating resources required for the ongoing use of existing telescopes and instrumentation. As a result, the Blanco telescope is transitioning to a complement of a few, permanently mounted, facility instruments that make best scientific use of its capabilities in our new multi-telescope environment. The Infrared Side Port Imager (ISPI) will be one of this complement, initially sharing the Ritchey-Chretien focus with the fiber-fed optical spectrograph HYDRA II.

ISPI's design choices have been shaped by an emphasis on imaging only, the restricted space envelope determined by the 4-m mirror cell above and the HYDRA fiber table below its bent-Cass location, the need to produce a new instrument rather quickly, and constrained capital and personnel resources. Schedule and budget have been major drivers for systems trades. We have relied heavily on in-house design heritage for mechanical design solutions, and commercial components for electronics and software. In the following sections we discuss the optical, mechanical, and thermal design, array control electronics, software, and status including initial laboratory characterization of our science grade HAWAII 2 array.

\section{OPTICAL DESIGN}

The ISPI optical design originated with a design produced by C. F. W. Harmer (NOAO) for the University of Florida IR camera FLAMINGOS $^{3}$. This existing design was a reasonable match to our science-driven specifications for field of view and per pixel sampling, was already optimized for the Mayall 4-m telescope, and offered considerable cost savings by a joint procurement of optics together with the FLAMINGOS project. Hence we adopted it as the baseline for ISPI. The Blanco 4-m has slightly different optical parameters, and we wished to operate with the first collimator lenscolder than in FLAMINGOS. This required a rebalancing of the element spacings in the collimator.

The optical design is shown in Figure 1. It is a refractive, collimator-camera system with filters at the intermediate cold pupil image. The $\mathrm{f} / 3$ camera employs one aspheric element, a field flattener just ahead of the detector. All other surfaces are spheres. We investigated the addition of cold-inserted auxiliary optics for reimaging the pupil onto the array. While this has telescope alignment and diagnostic advantages, the additional complexity and cost were unacceptable trades so this was not implemented. The scale on the Blanco 4-m is 0.325 arcsec per pixel, resulting in an 11 x 11 arcmin field of view. This design coupled to the telescope easily meets or exceeds a science-derived 2 pixel criterion for $80 \%$ encircled energy diameter with reasonable alignment tolerance allowances. Performance criteria and alignment tolerances are given in Tables 1 and 2.

As indicated on the right side of Figure 1, ISPI is fed by a tertiary fold mirror mounted on an existing movable stage in the telescope guider. Guiding can be done with the present uplooking guide probes viewing around the edges of this mirror. 


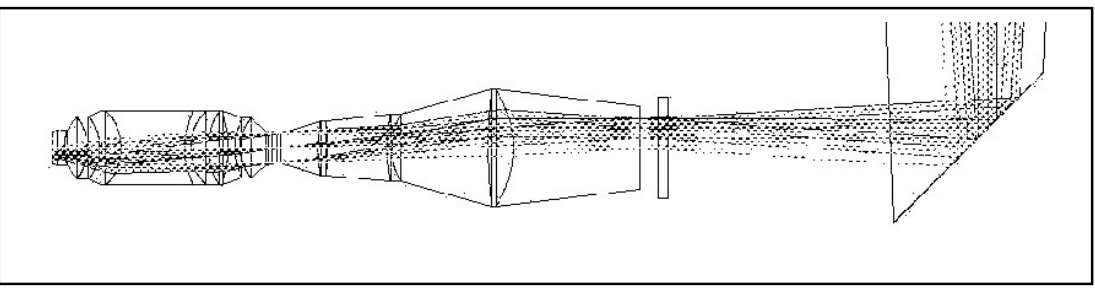

Figure 1. Ray trace of ISPI optical configuration, detector to the left.

\begin{tabular}{|c|c|c|c|c|}
\hline \multirow{2}{*}{ Band } & \multicolumn{2}{|c|}{$80 \%$ EE, arcsec } & \multicolumn{2}{|c|}{ Distortion } \\
\cline { 2 - 3 } & Best & Worst & Center-edge & Center-corner \\
\hline $\mathrm{J}$ & 0.36 & 0.48 & & \\
\hline $\mathrm{H}$ & 0.36 & 0.48 & \multirow{2}{*}{$1.13 \%$} & $0.77 \%$ \\
\hline $\mathrm{K}$ & 0.48 & 0.55 & & \\
\hline
\end{tabular}

\begin{tabular}{|c|c|c|c|}
\hline & $\begin{array}{l}\text { Tilt, } \\
\text { deg }\end{array}$ & $\begin{array}{l}\text { Decenter } \\
\mathrm{mm}\end{array}$ & $\begin{array}{l}\text { Despace } \\
\mathrm{mm}\end{array}$ \\
\hline $\begin{array}{l}\text { Individual elements } \\
\text { in groups }\end{array}$ & \pm 0.1 & \pm 0.07 & \pm 0.3 \\
\hline Collimator group & \pm 0.2 & \pm 0.2 & $\begin{array}{l} \pm 1 \text { wrt } \\
\text { camera }\end{array}$ \\
\hline Front camera group & \pm 0.1 & \pm 0.1 & \multirow{2}{*}{$\begin{array}{c} \pm 1 \text { between } \\
\text { groups }\end{array}$} \\
\hline Rear camera group & \pm 0.1 & \pm 0.1 & \\
\hline
\end{tabular}

Table 1. Imaging performance metrics for ISPI.

Table 2. Alignment tolerances for ISPI optics.

The optics were produced by Janos Technology, Inc. The materials are $\mathrm{BaF}_{2}, \mathrm{CaF}_{2}, \mathrm{ZnSe}$ and $\mathrm{ZnS}$. The polycrystalline nature of some of the fluoride lenses gives them a peculiar appearance in white light (Figure 2). The $65 \mathrm{~mm}$ diameter $J H K_{S}$ filters are being obtained from Barr Associates through a consortium purchase organized by Alan Tokunaga.

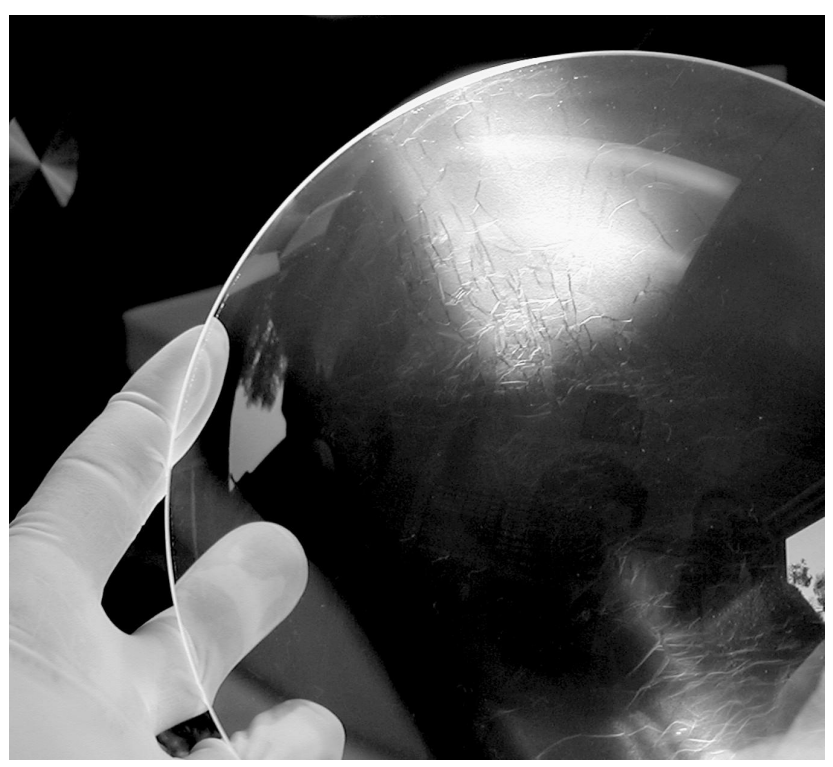

Figure 2. Large polycrystalline $\mathrm{BaF} 2$ collimator lens under strong edge illumination. The threadlike structures seen at top center and bottom right are in the bulk of the material and do not reach the surface. 


\section{MECHANICAL AND THERMAL DESIGN}

The array operating temperature requirement and internal thermal background constraints are both satisfied by operating the instrument at liquid nitrogen temperature. We chose liquid cryogens rather than closed cycle cooling for design and operational simplicity. The restricted available instrument envelope, a half cylinder of $\sim 1 \mathrm{~m}$ radius and $\sim 0.3 \mathrm{~m}$ thickness, did not favor an optics bench-plus-cryogen tank implementation. Instead, we built on the design heritage of $\mathrm{CIRIM}^{4}$, deploying the optics in straight-line tubular supports surrounded by annular cryogen tanks. Figures 3 and 4 illustrate the resulting cylindrical dewar assembly.

The need for filter wheels and associated drive mechanisms breaks the cylindrical symmetry at the pupil plane. ISPI is composed of two dewars with separate annular $\mathrm{LN}_{2}$ tanks. The front dewar contains the collimator and the filter wheel assembly, and the rear dewar houses the camera and detector. Each cryogen tank has reentrant fill and vent tubes to allow for changing orientation on the telescope. While each dewar can be closed and used separately, such as for laboratory tests of the array, at the telescope the two share a common vacuum envelope. A fixed cold pupil stop is located between two filter wheels in the collimator dewar. These wheels are driven on their perimeters by external motors. Changing filters requires removal of the camera dewar for access to the wheel assembly. While ISPI is intended for imaging only, the internal layout allowed preservation of some upgrade paths with no design or cost impact. A separate snout forms the vacuum seal to the cold telescope focal plane. This could be replaced, e.g. with a cold multislit assembly for spectroscopy. There is mechanical space ahead of the first filter wheel for thick elements such as grisms or polarizing elements.

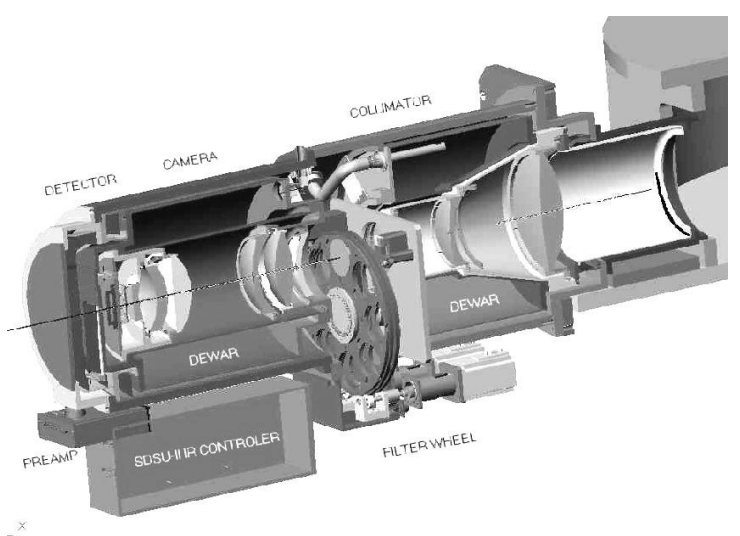

Figure 3. Cutaway with major components labeled.

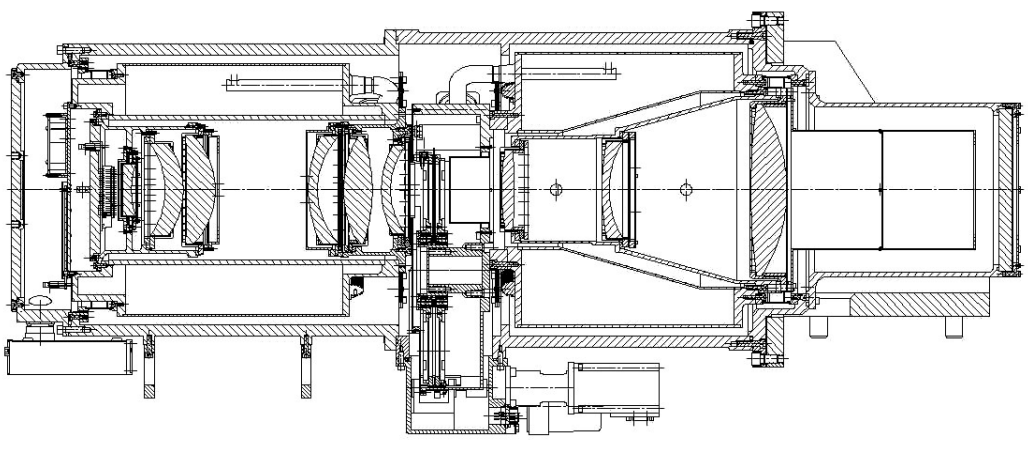

Figure 4. Side view mechanical drawing.

The collimator lens cells are mounted in a single machined housing that is surrounded by a separate cryogen tank. The camera cells are built up into subassemblies that insert into a machined tubular housing. This housing forms the inner wall of the rear cryogen tank.These housings are rigidly fixed to their dewars at the front and rear ends, respectively. Fiberglass flex fixtures at the ends near the cold pupil hold them rigidly in the transverse direction but allow for longitudinal thermal expansion. The optical design is tolerant to despace in this collimated portion. The emphasis throughout mechanical design, fabrication, and assembly has been to maintain tight optical alignment tolerances; no adjustments are incorporated. This is challenging with our two dewar design. The collimator and camera dewars must join with their optical axes coincident. Project engineer A. Montané worked closely with CTIO instrument makers during fabrication to insure that tolerances did not accumulate in a systematic fashion at this interface. The internal optics assemblies have been integrated and tested in a bench setup, sans dewars, and are found to satisfy alignment tolerances and warm optical performance checks as-built. After a final complete dewar integration and cold performance check, the system will be disassembled and interior surfaces coated with Aeroglaze ${ }^{\circledR}$ Z306 black ${ }^{5}$.

In order to accommodate differential thermal expansion between metal and optic, some cryogenic lens cell designs produce decentered optics when the system is warm. ISPI uses a "spring finger" cell with strong CTIO design heritage that preserves centration warm and cold. This makes warm optical performance verification easier. Figure 5 shows the cell design for the large $\mathrm{BaF}_{2}$ front collimator lens. At room temperature the lens is a slip fit into a slotted annular ring. Finger stiffness holds the lens 
weight under gravity in a horizontal orientation. Finger length is adjusted so that the radial pressure on the lens when cold is modest. Friction with whatever fingers are lateral in a given orientation is the chief means of preventing cold decenter. The lens is retained against despace by a springy stainless steel ring that presses a collar into place from the back. $\mathrm{BaF}_{2}$ is notoriously susceptible to mechanical and thermal stress. This design distributes the mechanical loads around the perimeter. Safely cooling the lens is a separate problem. From analytical formulae, thermal finite element analysis, and empirical instrumented cold tests of a dummy lens in this cell, we decided that conductive cooling of this thick convex lens through its thin edge posed an unacceptable risk. Fortunately it has a substantial view factor to cold, high emissivity surfaces in the blackened interior of the collimator assembly. Accordingly, the cell is thermally insulated with a fiberglass ring and the dominant cooling mechanism is radiation. This is very uniform over the lens surface. We expect cooling to a steady state temperature of $\sim 150 \mathrm{~K}$ in $\sim 25$ hours, which meets predetermined performance requirements. The remaining lenses use the same cell design, and reach colder temperatures more quickly. Being substantially smaller (and some of thermally tougher materials) these did not pose a cooling risk.

Thermal control of the array is described in the following section.
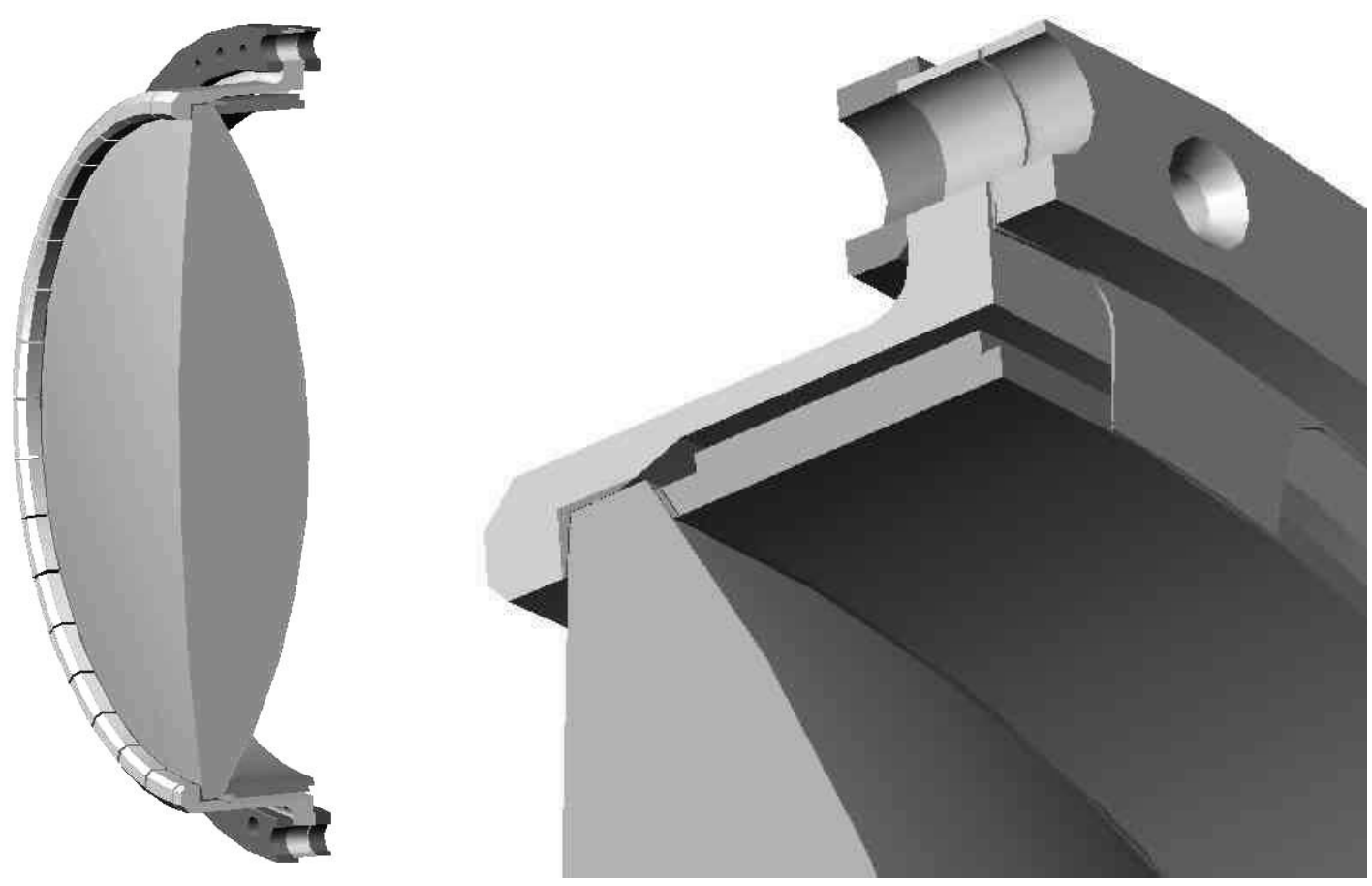

Figure 5. Spring finger lens cell for the large $\mathrm{BaF}_{2}$ collimator lens. Left, cross section showing annular ring cut to form fingers. Right, closeup showing a finger, retention collar loaded by tabs on a spring ring, and fiberglass ring for thermal isolation. 


\section{ELECTRONICS DESIGN}

ISPI uses a 2048 x 2048 Hawaii $2^{\mathrm{TM}} \mathrm{HgCdTe}$ array from Rockwell Scientific ${ }^{6}$. This was the largest format production array available at the start of the project. It has a strong legacy from $1 \mathrm{~K} \mathrm{x} 1 \mathrm{~K}$ and smaller arrays developed by this vendor. This choice also built on CTIO experience with implementing HgCdTe technology in previous instruments.

The array control electronics are required to provide flexible operations of the array, a frame rate fast enough to support $\mathrm{K}$ band imaging, and easy interfacing with the instrument control system. After investigating various options, including extension of the CTIO-developed ARCON controller, we decided to purchase a commercial controller and limit in-house effort to system integration and software development. The purchased solution is a Generation II controller from the San Diego State University CCD Laboratory (SDSU II) ${ }^{7}$, with IR video boards and a Hawaii 2 specific preamplifier provided by Infrared Laboratories ${ }^{8}$. CTIO had already chosen the SDSU II controller for the SOAR Optical Imager ${ }^{9}$, so there was a resource savings by piggybacking on that project's implementation effort. We also expect operational support savings from this commonality.

A system block diagram is shown in Figure 6 . The $\mathrm{K}$ band frame rate requirement, with 0.3 arcsec pixels on a $4 \mathrm{~m}$ telescope and $\sim 10^{5}$ electrons well capacity, is $\sim 3$ seconds. While the controller can be expanded to match the 32 output configuration of the array, we are implementing the 4 output configuration. This satisfies the frame rate requirement at a per pixel readout speed that does not degrade the output waveform, and is a considerable cost savings over the 32 channel alternative. The system noise requirement for the controller signal chain in our application is undemanding; 20 electrons rms, assuming array readout noise of 10 electrons rms. The dark current requirement, $<1$ electron/sec, is also modest. Electronics self-emission of the Hawaii 2 was stated to be much reduced compared to the Hawaii 1 by removal of some on-chip circuitry ${ }^{10}$. For these reasons we chose to use the on-chip amplifiers rather than external buffering, and the Infrared Laboratories single-ended preamp rather than a differential signal technique for the output signal. This design simplicity also kept electronics development costs low.

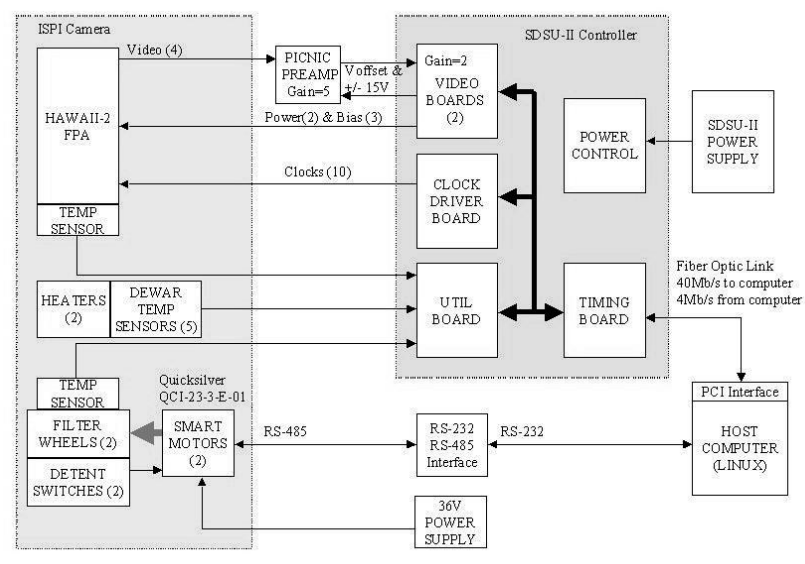

Figure 6. Array control electronics block diagram.

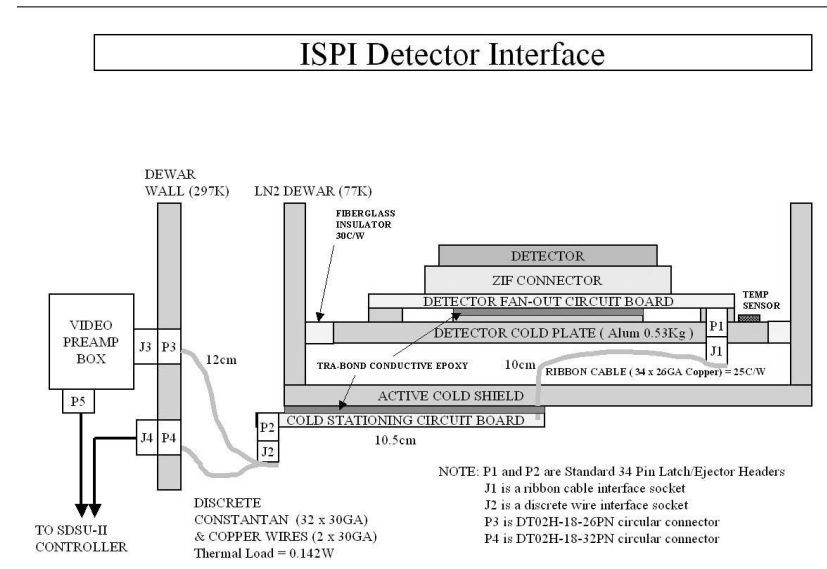

Figure 7. Schematic electrical and thermal detector interface.

Implementation of the vendor supplied electronics went smoothly. A capacitor was changed on the video clock driver card to speed up the pixel clocks. This put the rise time and overlap within Rockwell's specifications for the array. Additional gain was added to the preamp offset circuitry to allow array characterization over a wide range of biases. This also eliminated a low frequency interchannel coupling that degraded photometric accuracy as a function of image brightness and spatial frequency. The cable from the array to the video boards was modified to eliminate a connector and improve shielding. Unbroken shields and an absence of ground loop paths are important. We used signal and EMI shielding to minimize noise pickup throughout the design. Grounding options were limited by the internal configuration of the SDSU controller. We left as much flexibility as possible in the design, then used laboratory and on-telescope tests to guide implementation. The final grounding scheme consists of single point grounds. Analog and digital grounds are separate on the detector. The detector mount is isolated from the detector and 
floating with respect to the external dewar. The instrument is isolated from the telescope and connected to the external facility ground through the SDSU II power supply case.

Electronics inside the dewar are limited to a fanout board behind the detector, connectors, cables, and a cold stationing board to intercept the thermal load coming in via the wiring (Figure 7). The warm preamp plugs into a connector at the dewar wall. The detector mount assembly is very simple. The pin grid array ceramic chip carrier is held in a zero insertion force socket soldered to the fanout board. This in turn is epoxied to a large aluminum plate that caps the camera assembly. A jig was used to ensure precise mechanical centering for gluing. Any despace or tilt correction determined to be necessary for good uniform focus across the array will be done by remachining an interface ring between the end plate and the camera assembly. (Telescope refocus is the first corrective for despace error. The absence of mechanical structures such as slits at the first focal plane permits some freedom in its precise location.) The fanout board has eight layers: two analog and two digital signal layers plus four ground planes. It also carries pullup resistors for the video outputs and decoupling capacitors for bias voltage filtering.

The cold stationing board has three copper layers (analog, digital, and ground plane) and an uncoated fiberglass face epoxied to the active cold shield near the detector. This provides a thermally conductive path from the board lands to $\mathrm{LN}_{2}$. The array is cooled via the central $15 \times 15$ pins of the chip carrier through the socket and fanout board. It was found necessary to moderate the detector cooldown rate by adding a fiberglass ring at the interface to the rear camera assembly. The detector temperature is monitored with a high gain temperature sensor interface built at CTIO. We do not use active detector temperature control. Thermally induced drift is minimized by the detector mount time constant of $\sim 2$ hours and its intimate contact with the liquid cryogen. The initial design specification for detector temperature stability was $<0.1 \mathrm{~K} / \mathrm{hour}$ of drift, derived from the change of bias with temperature reported by the NIFS team for Hawaii 1 arrays ${ }^{11}$. Subsequent measurements with our Hawaii 2 engineering grade array showed much less bias drift with temperature. We believe our simple passive arrangement will be satisfactory. A heater is provided on the aluminum end plate of the detector assembly. This will be used to ensure that the detector does not function as a cryopump during warmup. It also provides a means to characterize the detector at elevated temperatures.

The filter wheels are driven by integrated AC servomotor assemblies from Quicksilver Controls ${ }^{12}$. These incorporate a programmable microprocessor which receives high level commands directly from the instrument computer. The filter motor power supply ground is left floating to prevent ground loops.

ISPI will be maintained cold on the telescope for long periods. A useful operations protocol for vacuum maintenance may be occasional warmup, just to the point of outgassing the getter, while pumping on the vacuum. This would renew the vacuum while minimizing thermal stress on the $\mathrm{HgCdTe} / m u l t i p l e x e r$ bond. Hence vacuum monitoring and logging are important. We are using a combination Pirani and cold cathode sensor with control/display unit from Pfeiffer Vacuum ${ }^{13}$. The display is at the instrument for convenience during on-telescope pumpout. Telemetry for remote readout and data logging at the astronomer's station uses a proportional DC voltage produced in the display unit, fed to a commercial A/D converter and RS- 485 serial output module ${ }^{14}$ and read via an instrument computer serial port. Necessary programming was done in LabVIEW. The same system will be used in the SOAR Optical Imager.

\section{INSTRUMENT CONTROL SOFTWARE}

The requirements on instrument control software are to provide array operation, data readout and storage; communicate with the telescope; communicate with instrument filter wheels; support some scripting capability for automation of observing procedures; and provide an astronomer-friendly interface. There has been a proliferation of instrument operating systems at groundbased telescopes, an issue that has surfaced at CTIO with the shared use of university-developed instruments as quasi-facility instruments. To control schedule and cost we wished to use existing software. To ease the subsequent maintenance burden we sought commonality with other instrument control applications supported by the same people at the Tololo + Pachon sites. ISPI will be used at the Blanco $4 \mathrm{~m}$ telescope, where large pieces of software written in $\mathrm{C}$ already exist for telescope communication and control. At the time of ISPI project start, the SOAR telescope had chosen National Instruments LabVIEW ${ }^{15}$ under RTLinux $^{16}$ as its development environment for observatory control. The telescope control system was contracted to Imaginatics, Inc. ${ }^{17,18,19}$. CTIO staff seconded to the SOAR Consortium were developing instrument control software for the SOAR Optical Imager (with its SDSU II controller) using ArcVIEW, a LabVIEW-based proprietary software package also produced by Imaginatics $^{20}$. ArcVIEW provides instrument control for all new SOAR instruments, including three under development by 
university groups ${ }^{21}$, and is also being used for new facility instruments on the Hale $5 \mathrm{~m}$ telescope (R. Smith, private communication). It is written in LabVIEW 6.0.2 and C, and hosted on PC workstations.

In order to piggyback on this effort, we chose ArcVIEW as the ISPI instrument control environment. ArcVIEW is a highly modularized system. A main application server provides communications and a command processor. It dynamically loads various modules that do the actual work. Modules are of three types: hardware-dependent (e.g. for filter wheel motor control), generic (e.g. a FITS image writer), and process-dependent. A process-dependent module follows the logical flow of a well-delimited (although possibly complex) process and may call on the other module types during execution. For example, the correlated double sampling process module calls on the controller and memory subtraction modules (hardware dependent) to take and difference two frames, then the unscrambler and FITS writer modules (generic) to form the image and write it to disk. ArcVIEW is extremely configurable and allows the addition of new features with little or no recoding or recompiling by creating appropriate modules. Further details are provided in a related paper at this meeting ${ }^{20}$.

The ISPI implementation has a great deal of commonality with the SOAR Optical Imager instrument control. The astronomer commands instrument functions with a GUI written in LabVIEW (Figure 8). LabVIEW enables a visually rich GUI development with an emphasis on "seeing" rather than "reading". Communication between the GUI and the main ArcVIEW application uses a LabVIEW based TCP/IP client/server implementation, the SOAR Communications Library. The external observatory components (telescope, guider, dome) already had a communications router and protocol written in C, so we created a LabVIEW module to interface between the main application and that router. A LabVIEW plugin was written for filter wheel control. Scripting of observing instructions to form automated sequences is supported in two ways, with IRAF cl scripts or via the ArcVIEW Gscript tool. This required development of an implementation task for IRAF-LabVIEW communications. Some "canned" scripts are provided, e.g. for an N x M raster with N, M, and telescope offset between positions as user-defined parameters. Throughout this development we have maintained the SOAR approach: LabVIEW serves as the "glue" between subsystems that themselves use whatever language is employed in pre-existing code, or is convenient or natural to their requirements.

\section{STATUS}

ISPI is presently (July 2002) undergoing final integration and laboratory cold tests. ISPI is scheduled for first light on the telescope in September 2002. Following a second commissioning run in November, it will begin scientific operation in January 2003. This is a delay of about 9 months from our original schedule due to external programmatic reasons. On two occasions the FLAMINGOS instrument fell victim to an accident resulting in the breakage of a lens. The ISPI project donated or loaned its identical lens until a replacement could be fabricated. Since FLAMINGOS was already scheduled for science operations at a variety of telescopes, keeping it on the air was regarded as a high priority for NOAO in the context of the U.S. system of astronomy facilities.

\section{ACKNOWLEDGEMENTS}

Other NOAO staff who have contributed substantially to this project are H. Ochoa, R. Rivera, F. Collao, M. Martinez, and R. Cantarutti (La Serena) and E. Pearson (Tucson). We thank our vendor contacts for their patient assistance: A. Haas and C. Cabelli (Rockwell Scientific), K. Salvestrini (Infrared Laboratories), R. Leach (San Diego State University), and D. Ballantine and D. Kaneshiro (Janos Technologies). The thorough open documentation regarding the same detector-controller combination on the NIFS website was very helpful to us.

\section{REFERENCES}

1. http://www.us-gemini.noao.edu/

2. http://www.soartelescope.org/

3. http://www.astro.ufl.edu/ elston/flamingos/home_main.html

4. http://www.ctio.noao.edu/instruments/ir_instruments/cirim/cirim.html

5. Manufactured by Lord Corporation, Chemical Products Division; http://www.lord.com/tradenames/Aeroglaze.htm

6. http://www.rsc.rockwell.com/imaging/hawaii2/index.html

7. http://mintaka.sdsu.edu/ccdlab/LabMain.html 
8. http://www.irlabs.com/cont.html

9. http://www.soartelescope.org/sac/OpticalImagerDesignReview/OpticalImager.html

10. C. Cabelli, private communication (reduction of glow on $\mathrm{H} 2$ vs $\mathrm{H} 1$ )

11. NIFS Conceptual Design Review documentation, Sec. 8.3.7.2, http://www.mso.anu.edu.au/ peter/nifs/codr/codr_detector.html

12. http://www.qcontrol.com/

13. Compact FullRange ${ }^{\mathrm{TM}}$ Gauge and SingleGauge ${ }^{\mathrm{TM}}$ Controller, http://www.pfeiffer-vacuum.com

14. D1000M Sensor to Computer Module, DGH Corp., http://www.dghcorp.com

15. National Instruments, http://ww.ni.com/

16. Finite State Machine Labs, Inc. ; http://www.fsmlabs.com/

17. Ashe, M. C., and Schumacher, G. "SOAR Telescope Control System: A Rapid Prototype and Development in LabVIEW", Proc. SPIE 4009, 48-60, 2000.

18. Ashe, M. C., Bonati, M., and Heathcote, S. "ArcVIEW: A LabVIEW-based Astronomical Instrument Control System", Proc. SPIE 4848, 2002 [this meeting]

19. http://www.imaginatics.net/

20. Schumacher, G., and Ashe, M. C. "SOAR TCS: From Prototype to Implementation", Proc. SPIE 4848, 2002 [this meeting]

21. Cecil, G. "First Generation Instruments for the SOAR 4.25m Telescope", Proc. SPIE 4008, 83-93, 2000

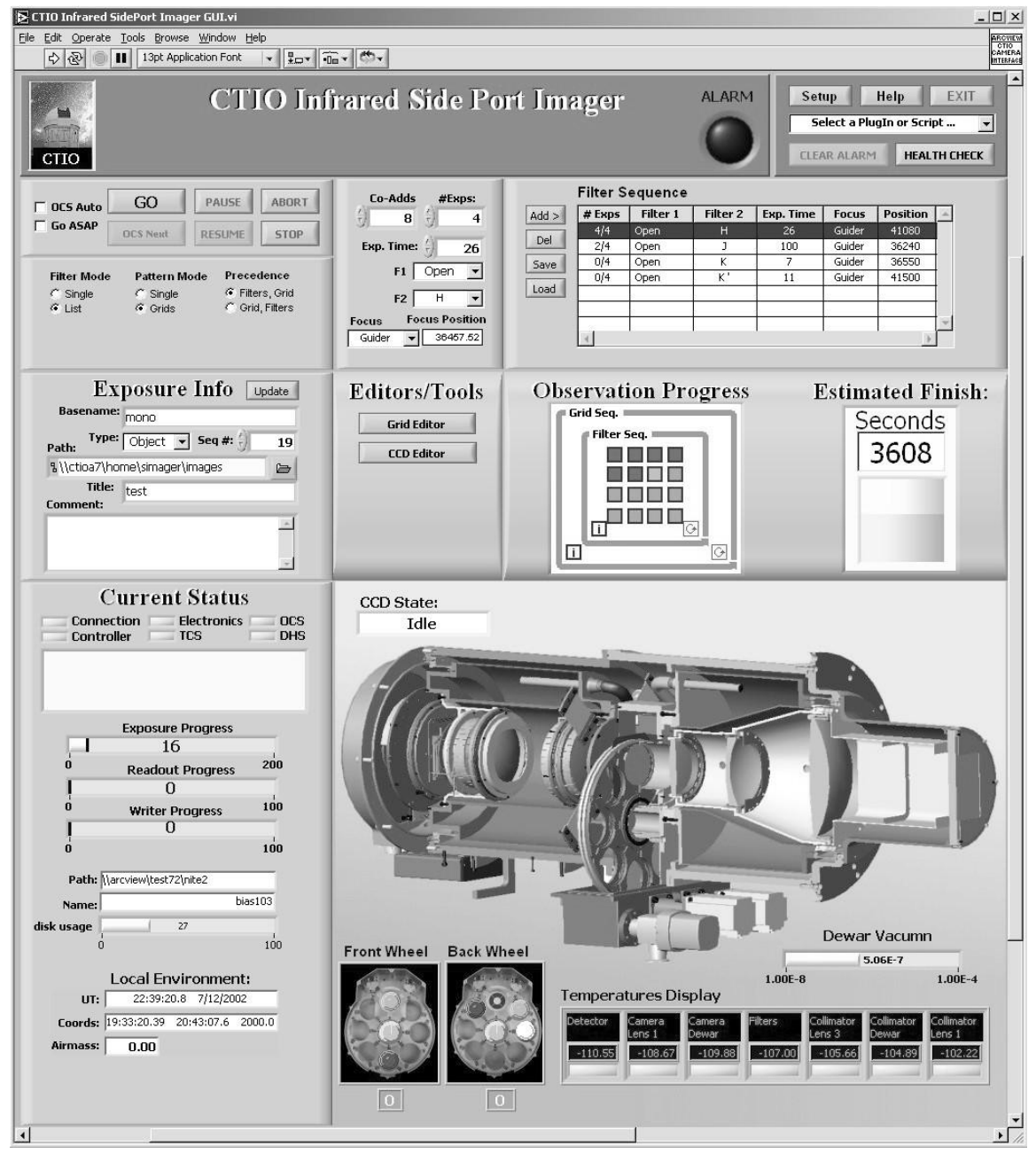

Figure 8. The ISPI astronomer user's GUI 